\title{
The Crossed Flexor Plantar Response in Patients with Klippel-Feil Syndrome
}

\author{
Anna Sadnicka Simon F. Farmer \\ The National Hospital for Neurology and Neurosurgery, London, UK
}

\section{Keywords}

Klippel-Feil syndrome $\cdot$ Flexor plantar response $\cdot$ Plantar reflex $\cdot$ Mirror movements

\begin{abstract}
The plantar reflex is one of most important and widely tested components of the neurological examination. We describe 3 subjects with Klippel-Feil syndrome and mirror movements where unilateral cutaneous stimulation of the foot leads to flexor plantar responses in both feet. We discuss the evidence which suggests that this "crossed flexor" plantar response reveals a transcortical pathway for the flexor plantar response.

(C) 2017 The Author(s) Published by S. Karger AG, Basel
\end{abstract}

\section{Background}

Klippel-Feil syndrome is a rare skeletal anomaly characterised by abnormal fusion of two or more vertebrae and the clinical triad of a short neck with restricted movement and a low posterior hairline (Fig. 1a) [1, 2]. Sporadic and genetic forms (autosomal dominant, recessive, and $\mathrm{x}$-linked) are described and the syndrome is most commonly diagnosed prenatally or at birth [3].

Klippel-Feil syndrome is associated with mirror movements by which voluntary unilateral movement of a limb causes involuntary movement or mirroring of the homologous muscles of the opposite limb [4,5]. Mirror movements are most often observed in the hands but can also be seen in distal lower limb musculature [6]. Magnetic resonance imaging (MRI) of 
the cervicomedullary junction of such patients demonstrates varying degrees of neuroschisis (posterior cleft through to complete neuroschisis), and increasing neuroschisis is associated with more prominent mirror movements [6] (Fig. 1b).

In this paper, we describe a novel physical sign seen in patients with Klippel-Feil syndrome who have mirror movements: the crossed flexor plantar response. In 3 patients, unilateral mechanical stimulation of the foot produced bilateral flexor plantar responses. We discuss how this novel clinical sign may shed further light on the anatomical pathway of the flexor plantar response in health.

\section{Case Presentation}

Three female patients (aged 39, 45, and 45 years) with Klippel-Feil syndrome were seen at the National Hospital for Neurology and Neurosurgery and St Mary's Hospital, London, UK. Written informed consent was obtained from the patients for the images and videos to be published. All patients had mirror movements of the fingers and toes. For example, 1 patient reported that when practising the piano if rehearsing 1 hand she would sit on the other hand to stop mirror movements from occurring. On examination, deep tendon reflexes were present and not crossed. During plantar response, testing subjects were asked to relax and to avoid voluntary movement during testing. Both right and left feet were stimulated at different times and the bilateral flexor plantar response sign was seen in all 3 subjects regardless of whether the right or left foot was stimulated. This is exemplified in a series of photo stills (Fig. 2) and in the online supplementary Video (see www.karger.com/doi/10.1159/000474935).

\section{Discussion}

The plantar response is a critical component of the neurological examination. The eponymous positive Babinski sign signifies disturbance of the pyramidal tract by upward movement of the great toe [7]. Implicit in our understanding is a belief that in order to produce a "healthy" flexor plantar response, the intact central nervous pathways exert control over a more primitive extensor plantar. This is supported by the observation that children, before they become ambulant, exhibit an extensor plantar response that is then "corticalised" by maturation of central motor pathways. Whilst these models of motor physiology make intuitive sense, they do not tell us with precision the pathways implicated in the plantar response, and whether the plantar reflex is solely spinal or transcortical has been the subject of much debate [8]. We discuss evidence that the crossed flexor plantar response in KlippelFeil syndrome with mirror movements may shed further light on the neuroanatomy of the plantar reflex pathway.

In healthy individuals, transcranial magnetic or electrical stimulation of the motor cortex typically elicits muscle contraction in the opposite side of the body only. In patients with Klippel-Feil syndrome and mirror movements, stimulation to either hemisphere evokes bilateral simultaneous responses [9]. The short latency of the unilateral and contralateral motor evoked responses (approximately $20 \mathrm{~ms}$ ) is fully compatible with corticospinal conduction, and the ipsilateral responses are thought to be mediated by an anomalous uncrossed corticospinal pathway [9]. Furthermore, when electromyography (EMG) is used to study mirror movements, cross-correlation analysis of the simultaneous left and right muscle ac- 


\section{Case Reports in Neurology}

tivity reveals that there is short-term motor unit synchrony between homologous muscle pairs [9]. This is not observed in non-mirroring subjects and is indicative of abnormal common monosynaptic input to the left and right motor neurone pools in Klippel-Feil syndrome [9]. The hypothesis of aberrant corticospinal tracts is further supported by abnormalities of pyramidal decussation found on the autopsy of a person with Klippel-Feil syndrome [10].

These abnormal anatomical features in Klippel-Feil syndrome have therefore been used as a model by which to probe the neuroanatomical basis of motor phenomena observed in healthy subjects. For example, in Klippel-Feil syndrome during unilateral stimulation of stretch and cutaneous afferent pathways, there is crossing of the long-latency stretch and long-latency cutaneomuscular reflexes without crossing of short-latency components of the reflexes $[9,11]$. As the bilateral long-latency responses are remarkably symmetrical in their temporal and amplitude characteristics with a similar muscle distribution and behaviour, these neurophysiological observations revealed that these later components of the reflex are transcortical $[9,11]$. Our observation detailing an abnormally crossed flexor plantar response in 3 patients therefore provides strong evidence that their plantar response is also mediated by a transcortical reflex. To date, there is no evidence of an abnormally organised somatosensory pathway [9] in these patients.

Generalisation of our interpretation of this result to the flexor plantar response observed in neurologically intact adult subjects without mirror movements is difficult given that the Klippel-Feil syndrome subjects have highly abnormal neuro-anatomy at the brainstem and cervical levels. However, the plantar responses we observed are qualitatively the same as those seen in normal subjects. Furthermore, our findings fit well with what is known about the plantar response, i.e., that it becomes flexor during motor development as spinal reflex pathways become subject to cortical control and that damage to the motor cortex and/or the descending white matter tracts leads to "release" of the reflex - the extensor plantar response or Babinski sign.

It is unusual to be able to describe a new physical sign, albeit in an unusual yet informative group of patients. Careful consideration of the nature of the crossed plantar reflex in patients with Klippel-Feil syndrome and mirror movements may be used to inform our understanding of the mechanisms that underlie one of the commonest and most important parts of the neurological examination, the flexor plantar reflex.

\section{Acknowledgements}

We would like to thank the patients included in this study. We thank Dr. Jonathon Frankel for referring one of the cases and Dr. John Stevens for reviewing the radiology.

\section{Statement of Ethics}

Written informed consent was obtained from the patients for the images and videos to be published. 


\section{Case Reports in Neurology}

\section{Disclosure Statement}

On behalf of all authors, the corresponding author states that there are no conflicts of interest.

\section{References}

1 Noble TP, Frawley JM: The Klippel-Feil syndrome: numerical reduction of cervical vertebrae. Ann Surg 1925;82:728-734.

2 Saker E, Loukas M, Oskouian RJ, Tubbs RS: The intriguing history of vertebral fusion anomalies: the Klippel-Feil syndrome. Childs Nerv Syst 2016;32:1599-1602.

-3 Giampietro PF, Raggio CL, Blank RD, McCarty C, Broeckel U, Pickart MA: Clinical, genetic and environmental factors associated with congenital vertebral malformations. Mol Syndromol 2013;4:94105.

-4 Bauman GI: Absence of the cervical spine: Klippel-Feil syndrome. J Am Med Assoc 1932;98:129-132.

-5 Baird PA, Robinson GC, Buckler WS: Klippel-Feil syndrome. A study of mirror movement detected by electromyography. Am J Dis Child 1967;113:546-551.

-6 Royal SA, Tubbs RS, D’Antonio MG, Rauzzino MJ, Oakes WJ: Investigations into the association between cervicomedullary neuroschisis and mirror movements in patients with Klippel-Feil syndrome. AJNR Am J Neuroradiol 2002;23:724-729.

7 Babiński JJFF: Sur le réflexe cutané plantaire dans certaines affections organiques du système nerveux central. C R Seanc Soc Biol 1896;3:207-208.

8 Landau WM, Clare MH: The plantar reflex in man, with special reference to some conditions where the extensor response is unexpectedly absent. Brain 1959;82:321-355.

-9 Farmer SF, Ingram DA, Stephens JA: Mirror movements studied in a patient with Klippel-Feil syndrome. J Physiol 1990;428:467-484.

10 Gunderson C, Solitare G: Mirror movements in patients with the Klippel-Feil syndrome. Neuropathological observations. Arch Neurol 1968;18:675-679.

11 Matthews PB, Farmer SF, Ingram DA: On the localization of the stretch reflex of intrinsic hand muscles in a patient with mirror movements. J Physiol 1990;428:561-577. 


\section{Case Reports in Neurology}

\begin{tabular}{l|l}
\hline Case Rep Neurol 2017;9:143-148 \\
\hline DOI: 10.1159/000474935 & $\begin{array}{l}\text { C 2017 The Author(s). Published by S. Karger AG, Basel } \\
\text { www.karger.com/crn }\end{array}$ \\
\hline
\end{tabular}

Sadnicka and Farmer: The Crossed Flexor Plantar Response in Patients with Klippel-Feil Syndrome
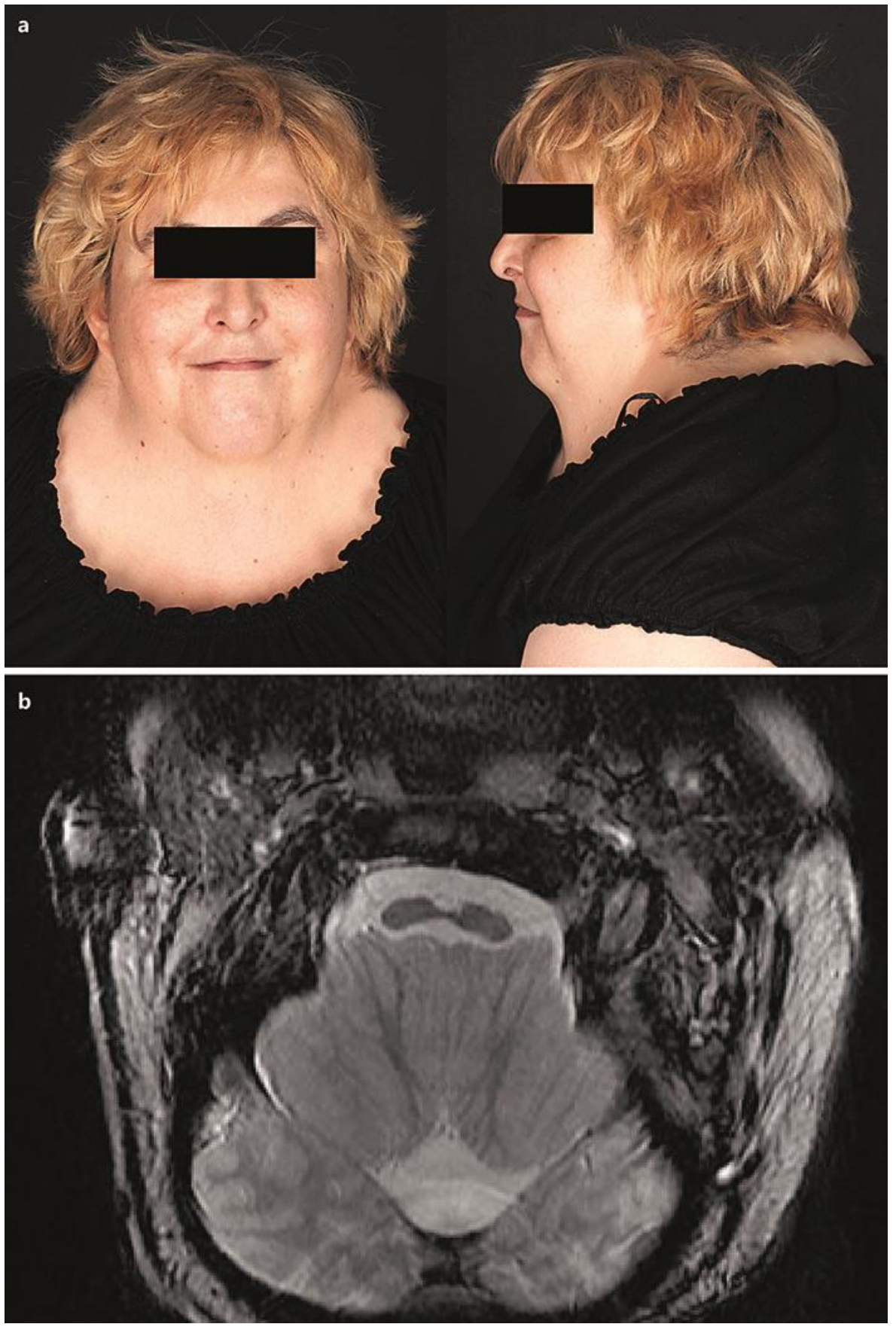

Fig. 1. a The clinical triad of Klippel-Feil syndrome; a short neck, low hairline, and limited neck mobility. b Axial MRI image at the level of the medullary pyramids shows typical cervicomedullary neuroschisis. In this subject, the cleft extended caudally to the levels at which the cervical roots emerged. 


\section{Case Reports in Neurology}

(C) 2017 The Author(s). Published by S. Karger AG, Basel

Sadnicka and Farmer: The Crossed Flexor Plantar Response in Patients with Klippel-Feil Syndrome
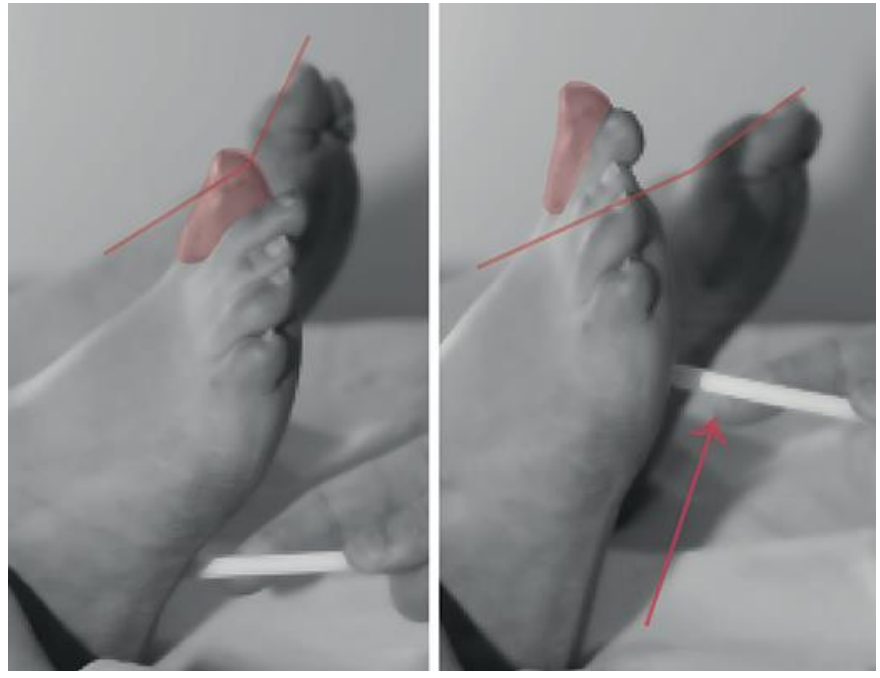

Fig. 2. The crossed flexor plantar response. Stills from the online supplementary Video show flexion in both toes after sensory stimulation to the right foot only. 